\title{
Gene profiling of human fetal and adult adrenals
}

\author{
W E Rainey, B R Carr, Z-N Wang and C R Parker Jr ${ }^{\mathbf{1}}$ \\ Department of Obstetrics and Gynecology, Division of Reproductive Endocrinology, University of Texas, Southwestern Medical Center, Dallas, \\ Texas 75390, USA \\ ${ }^{1}$ Department of Obstetrics and Gynecology, University of Alabama at Birmingham, Birmingham, Alabama 35294, USA \\ (Requests for offprints should be addressed to W E Rainey)
}

\begin{abstract}
The mechanisms that lead to the steroidogenic differences in the human fetal adrenal (HFA) and adult adrenal gland are not known. However, gene expression clearly plays a critical role in defining their distinct steroidogenic and structural phenotypes. We used DNA microarrays to compare expression levels of several thousand transcripts between the HFA and adult adrenal gland. Total RNA was isolated from 18 HFA and 12 adult adrenal glands. Samples of total RNA were used to make five pools of poly A+ RNA (mRNA). Gene profiling was done using five independent microarrays that contained between 7075 and 9182 cDNA elements. Sixty-nine transcripts were found to have a greater than $2 \cdot 5$-fold difference in expression between HFA and adult adrenals. The largest
\end{abstract}

differences were observed for transcripts that encode IGF-II (25-fold higher in HFA) and 3 $\beta$-hydroxysteroid dehydrogenase (24-fold higher in adult). Among the other genes, transcripts related to sterol biosynthesis or to growth and development were higher in the HFA than adult adrenals. Transcripts concerned with cellular immunity and signal transduction were preferentially expressed in the adult adrenal. The vast majority of the 69 transcripts have not been studied with regard to adrenal function. Thus, these gene profiles provide valuable information that could help define the mechanisms that control adrenal function.

Journal of Endocrinology (2001) 171, 209-215

\section{Introduction}

The human adrenal gland plays important, albeit different, roles during fetal development and adult life. There are major structural differences between the mid-gestational human fetal adrenal (HFA) and the adult adrenal gland. For example, during most of gestation, the HFA is characterized by a large inner cortical zone, the fetal zone that is surrounded by a thin layer of cells known as the neocortex (Mesiano \& Jaffe 1997). In contrast, the adult adrenal is composed of three morphologically distinct cortical zones and a well-defined medulla. In addition, it has been demonstrated that there are substantial differences in the endocrine functions of the HFA and adult adrenals. The adult adrenal secretes aldosterone as well as large quantities of both cortisol and dehydroepiandrosterone (DHEA) sulfate, while the mid-gestational HFA secretes substantial amounts of DHEA sulfate but minimal amounts of cortisol or aldosterone. These basic differences in function have long been suspected to result from alterations in gene expression.

Interestingly, the expression of only a few genes has been directly compared between the HFA and adult adrenals. The differences in expression for two such genes, insulin-like growth factor-II (IGF-II) (Mesiano et al. 1993,

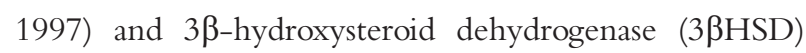
(Doody et al. 1990, Voutilainen et al. 1991), has been useful in explaining some of the functional differences between the HFA and adult adrenals. A more global understanding of gene expression in the HFA and adult adrenal gland would provide new insights into the functional differences between these stages of adrenal development. In the present investigation, we sought to identify the genes that are differentially expressed in the HFA and adult adrenal gland using DNA microarray technologies.

\section{Materials and Methods}

\section{Adult and fetal adrenal tissues}

Human adult adrenal glands were obtained at the time of nephrectomy for renal carcinoma and were determined to be normal at the time of pathological examination. HFA were obtained at the time of pathological examination of abortive tissue between 15 and 20 weeks of gestation. Adrenal glands were snap frozen in liquid nitrogen and stored at $-80{ }^{\circ} \mathrm{C}$ until use. The use of these tissues was approved by the Institutional Review Board of the University of Alabama at Birmingham. 


\section{RNA extraction}

Total RNA was extracted from tissues as previously described (Freije et al. 1997). Adrenals were pulverized in liquid nitrogen and then homogenized in guanidinium isothiocyanate. Purity and integrity of the RNA were checked spectroscopically and by gel electrophoresis prior to use. For array analysis, total RNA was used to isolate poly A+ RNA using two successive oligo (dT) cellulose columns and following the manufacturer's directions (Amersham Pharmacia Biotech, Piscataway, NJ, USA).

\section{Northern analysis}

Samples of total RNA $(10 \mu \mathrm{g})$ were separated by electrophoresis on $1 \%$ agarose gels in the presence of formaldehyde. RNA was transferred to a nylon membrane (Amersham) by overnight blotting at $10 \mathrm{~V}$ and was crosslinked under UV light. Hybridizations were performed at $42{ }^{\circ} \mathrm{C}$ for $16 \mathrm{~h}$, using cDNA probes. RNA probing was accomplished using cDNA fragments from $3 \beta \mathrm{HSDII}$ (GenBank accession M77144, nucleotides 7907-8080), nerve growth factor-inducible B (NGFIB) (GenBank accession NM002135, nuceleotides 1929-2311), KIAA0018 (GenBank accession AF261758, nucleotides 63-1648) and IGF-II (GenBank accession S77035, nucleotides 48-349). RNA hybridization with a cDNA probe against glyceraldehyde-3-phosphate dehydrogenase transcript was used to ensure RNA integrity and equal loading of lanes (data not shown).

\section{cDNA microarrays}

cDNA probe synthesis, hybridization with the UniGEM $\mathrm{V}$ microarray Version $1 \cdot 0$ (two times) and Version $2 \cdot 0$ (three times) and the signal analysis were conducted by Incyte Genomics (St Louis, MO, USA) as described at the company website, HTTP://www.incyte.com. The DNA microarray for Version 1 consisted of 7075 and the Version 2 consisted of 9182 unique probes arrayed onto glass slides. Isolated HFA and adult adrenal poly A + mRNA (200 ng) was reverse transcribed with the Cy5 (adult adrenal) or Cy3 (HFA) flourescent probes (Peron Technologies, Alameda, CA, USA). The paired reactions for both the HFA and adult adrenals were combined and purified. The two fluorescently labeled samples were simultaneously applied to each array. Following incubation, the microarray was rinsed and scanned using $532 \mathrm{nM}$ for $\mathrm{Cy} 3$ and then at $635 \mathrm{nM}$ for Cy5. A signal intensity of greater than 2.5-fold over background was used for data analysis. The ratio of the two fluorescent intensities was used as a quantitative measurement of the relative gene expression between the two tissue samples. Ratios from the five microarrays analysis were pooled to determine the mean \pm S.E.M.

\section{Results and Discussion}

RNA was isolated from a total of 18 HFA glands and 12 adult adrenal glands and was used on five genomic expression arrays. On average, $98 \%$ of the genes examined were expressed at a level that exceeded $2 \cdot 5$-fold that of background signal. A plot of all the gene comparisons from a representative array analysis is displayed in Fig. 1 with all transcripts with a greater than 2-fold difference represented by red dots. The vast majority of genes tested were expressed to a similar degree in the HFA and adult adrenals. Indeed, we found in three separate analyses using the UniGEM Version 2 microarray that only $1 \cdot 08 \%(100$ transcripts), $0 \cdot 88 \%$ (81 transcripts) and 2.65\% (243 transcripts) of the arrayed probes were expressed at levels that differed at least 2.5-fold between the HFA and adult adrenal RNA pools. In two separate analyses using the UniGEM Version 1 microarrays, there were 1.47\% (104 transcripts) and $2.58 \%$ (183 transcripts) of the arrayed elements with expression that differed by at least $2 \cdot 5$-fold between the HFA and adult adrenal tissue pools. From the five microarray analyses, 40 transcripts were determined to have an average expression of $>2.5$-fold intensity in the HFA than in the adult adrenal (Table 1). The transcript whose expression was most prevalent in the fetus compared with the adult was IGF-II. On the other hand, 29 transcripts were consistently expressed at $>2 \cdot 5$-fold intensity in the adult adrenal than in the HFA (Table 2). The transcript that was most prevalent in the adult compared with the fetus was $3 \beta \mathrm{HSD}$. The UniGEM Version 2 microarray had probes listed for $3 \beta \mathrm{HSD}$ type 1 and $3 \beta$ HSD type 2 . However, sequence analysis revealed that the similarity between the two probes would not allow specific identification of either transcript. This point emphasizes the need to examine closely the probes used on microarrays for gene families with a high degree of similarity. Transcript levels for IGF-II and 33HSD are recognized to differ between the HFA and adult adrenals (Doody et al. 1990, Voutilainen et al. 1991, Mesiano et al. 1993, 1997). The fluorescence scan for Cy5 (HFA) and Cy3 (adult adrenal) showed substantially greater expression of IGF-II in the HFA and strikingly enhanced expression of $3 \beta \mathrm{HSD}$ in the adult adrenal (Fig. 2). The marked differential expression of these genes was also evident upon traditional Northern analyses using $10 \mu \mathrm{g}$ total RNA from each tissue (Fig. 2).

Northern analysis was further used to compare the expression of two transcripts that have not previously been studied in fetal vs adult adrenals. NGFIB is an orphan nuclear receptor that appears to be an important regulator of transcription of certain of the steroid-metabolizing enzymes (Wilson et al. 1993). In addition, studies in mouse adrenal have demonstrated that high expression of NGFIB in the adult adrenal cortex is under the control of adrenocorticotropin (Davis \& Lau 1994). Both microarray and Northern analysis demonstrate that this transcription 
Table 1 Transcripts expressed at $\geq 2 \cdot 5$-fold higher levels in fetal vs adult adrenals

\section{Transcripts}

\section{Function}

Cell signalling

Sterol, steroid, or isoprenoid metabolism

Growth and development

Localized and structural protein

Other
Naturiuretic peptide receptor $\mathrm{C} /$ guanylate cyclase C

15-Hydroxyprostaglandin dehydrogenase

3-Hydroxy-3-methylglutaryl-coenzyme A reductase

lamosterol 14- $\alpha$-demethylase (CYP51)

Farnesyl-diphosphate farnesyltransferase 1

3-Hydroxy-3-methylglutaryl-coenzyme A synthase

Farnesyl diphosphate synthase

Sterol-C4-methyl oxidase-like

7-Dehydrocholesterol reductase

Mevalonate decarboxylase

Cholesterol side chain cleavage (CYP11A1)

IGF-II (somatomedin A)

IGF-II mRNA-binding protein-3

Cyclin-dependent kinase inhibitor IC (p57, Kip2)

Catenin (cadherin-associated protein), $\beta 1$ (88-kDa)

Midkine (neutrite growth-promoting factor-2)

p53 inducible protein

Fibroblast growth factor receptor 4

Cyclin-dependent kinase 8

Collagen, type I, $\alpha 1$

Fibrillin 2 (congenital contractural arachnodactyly)

Fibrinogen, A $\alpha$ polypeptide

Collagen, type $\mathrm{XI}, \alpha 1$

Kinesin heavy chain member 2

Microtubule-associated protein

Hemoglobin, $\gamma \mathrm{G}$
Osteoblast specific factor 2 (fasciclin I-like)
Butyrylcholinesterase
EST (uncharacterized transcript)
Deleted in liver cancer 1
Tetraspan 5
Galactosylceramidase (Krabbe disease)
3'-phosphoadenosine $5^{\prime}$-phosphosulfate synthase 2
P311 protein
KIAA0018 (uncharacterized transcript)
Deafness autosomal dominant 5
$26 S$ proteasome-associated pad 1 homolog
ATP citrate lyase
LM1955 H105e3 gene, partial cds
Galactosidase, $\beta 1$

Osteoblast specific factor 2 (fasciclin I-like)

Butyrylcholinesterase

ET (uncharacterized transcript)

Tetraspan 5

Galactosylceramidase (Krabbe disease)

3'-phosphoadenosine 5'-phosphosulfate synthase 2

P311 protein

KIAA0018 (uncharacterized transcript)

Deafness autosomal dominant 5

ATP citrate lyase

Galactosidase, $\beta 1$
Fold difference

\begin{tabular}{|c|c|}
\hline Mean & S.E. \\
\hline $6 \cdot 0$ & $1 \cdot 4$ \\
\hline $12 \cdot 0$ & $2 \cdot 9$ \\
\hline $7 \cdot 2$ & $1 \cdot 1$ \\
\hline $7 \cdot 1$ & $1 \cdot 3$ \\
\hline $4 \cdot 0$ & $0 \cdot 7$ \\
\hline $3 \cdot 8$ & 0.5 \\
\hline $3 \cdot 3$ & $0 \cdot 2$ \\
\hline $3 \cdot 2$ & $0 \cdot 2$ \\
\hline $3 \cdot 2$ & 0.4 \\
\hline $3 \cdot 0$ & $0 \cdot 4$ \\
\hline $2 \cdot 8$ & $0 \cdot 3$ \\
\hline $25 \cdot 0$ & $6 \cdot 7$ \\
\hline $5 \cdot 0$ & $1 \cdot 3$ \\
\hline $4 \cdot 1$ & $0 \cdot 3$ \\
\hline $3 \cdot 9$ & 0.7 \\
\hline $3 \cdot 5$ & $0 \cdot 3$ \\
\hline $3 \cdot 5$ & $0 \cdot 1$ \\
\hline $3 \cdot 4$ & $0 \cdot 3$ \\
\hline $2 \cdot 7$ & $0 \cdot 1$ \\
\hline $10 \cdot 2$ & $2 \cdot 7$ \\
\hline $5 \cdot 3$ & $1 \cdot 4$ \\
\hline $4 \cdot 6$ & $1 \cdot 3$ \\
\hline $3 \cdot 9$ & 0.5 \\
\hline $3 \cdot 0$ & 0.5 \\
\hline $2 \cdot 8$ & $0 \cdot 1$ \\
\hline $23 \cdot 0$ & $8 \cdot 7^{*}$ \\
\hline $9 \cdot 3$ & $1 \cdot 2$ \\
\hline $7 \cdot 5$ & $2 \cdot 1$ \\
\hline $6 \cdot 6$ & $1 \cdot 4$ \\
\hline $5 \cdot 4$ & $1 \cdot 7$ \\
\hline $5 \cdot 0$ & $1 \cdot 8^{*}$ \\
\hline $4 \cdot 8$ & $0 \cdot 6$ \\
\hline 4.5 & $1 \cdot 2$ \\
\hline $4 \cdot 1$ & $0 \cdot 4$ \\
\hline $4 \cdot 0$ & 0.9 \\
\hline $3 \cdot 8$ & $0 \cdot 8$ \\
\hline $3 \cdot 6$ & $0 \cdot 9$ \\
\hline $3 \cdot 2$ & $0 \cdot 3$ \\
\hline $3 \cdot 0$ & $0 \cdot 2$ \\
\hline $2 \cdot 8$ & $0 \cdot 3$ \\
\hline
\end{tabular}

*Indicates circumstances where the mean activity of the gene in the adult adrenal fell between the 95th and the 99th percentile confidence interval of that in the fetal adrenal. All others fell outside the 99 th percentile confidence interval for the fetal adrenal.

factor exhibits much higher expression in the adult than fetal adrenals (Fig. 2 and Table 2). Further study could define if this factor plays a role in the altered differentiation seen in the adult vs fetal adrenals.

KIAA0018 is a transcript that was recently shown to have altered expression in brains from Alzheimer's patients; however, its function is currently not known (Greeve et al. 2000). KIAA0018 was expressed at four times higher levels in the HFA when examined using either microarray or Northern analysis (Fig. 2). In addition, the signal intensity observed for KIAA0018 suggests that it is one of the most highly expressed transcripts in the HFA (Fig. 1). GenBank searches indicate that the only protein with structural similarity to KIAA0018 is the plant enzyme, DIMINUTO/ DWARF1, which is involved in plant steroid biosynthesis and critical for plant reproduction (Klahre et al. 1998). The role of this highly expressed transcript and its protein in the fetal adrenal will warrant further study.

The ability of the adult adrenal to produce aldosterone and cortisol requires the expression of aldosterone synthase (CYP11B2), 11ß-hydroxylase (CYP11B1), 21- 


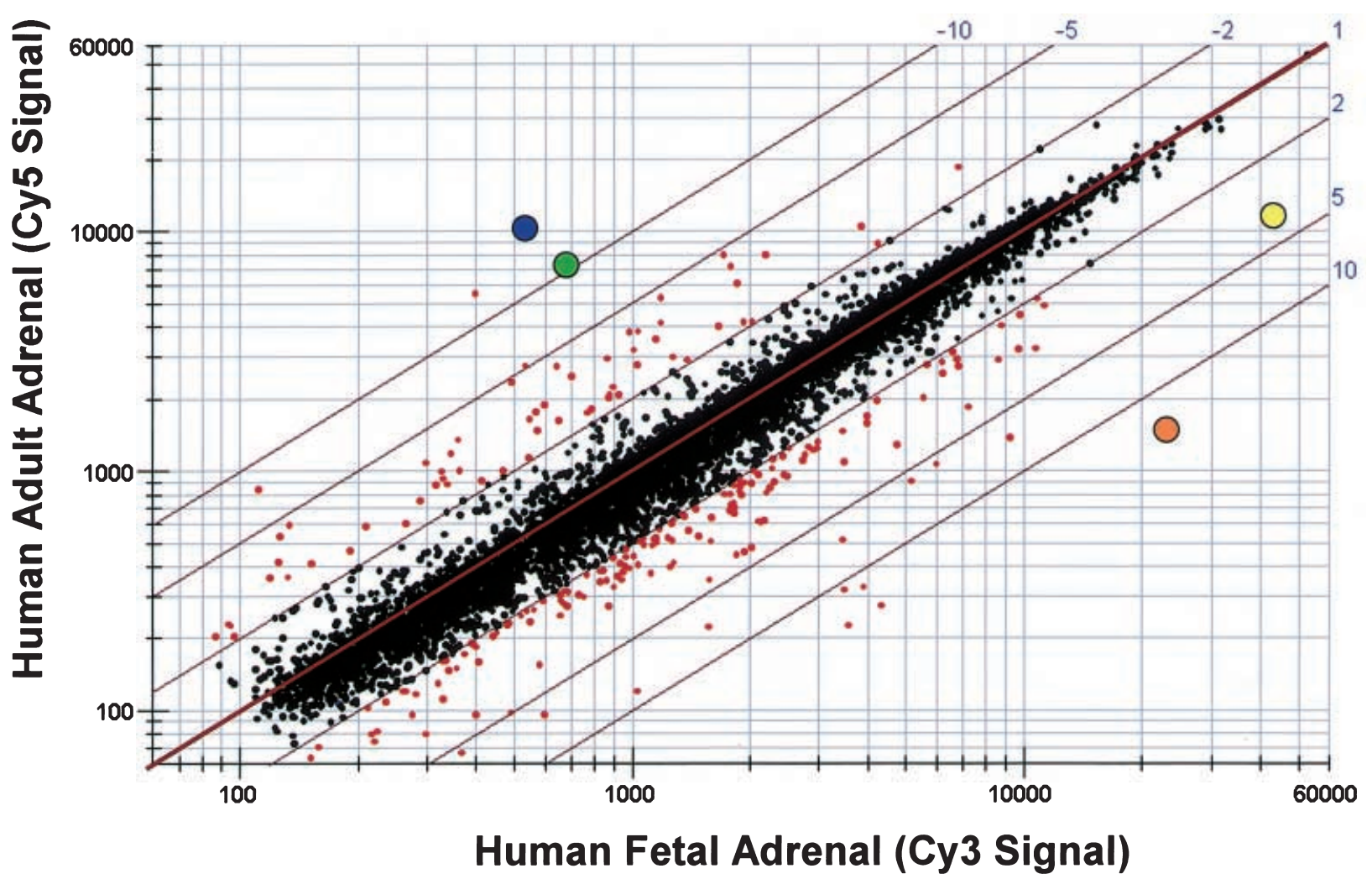

Figure 1 Microarray analysis comparing the mRNA expression patterns in human fetal and adult adrenal glands. Each spot represents a unique transcript with a total of 9182 genes examined on this array. Black dots represent transcripts with less than a 2-fold difference in expression, and red dots those with greater than 2-fold difference. 3ßHSD (blue spot), NGFIB (green spot), KIAA0018 (yellow spot) and IGF-II (orange spot) were further examined in Fig. 2.

hydroxylase (CYP21) and 3ßHSD. The similarity between the transcripts for the CYP11B1 and CYB11B2 isozymes should not allow the determination of individual expression levels by microaarray. However, the adult adrenal expressed 2:0 $\pm 0 \cdot 2$-fold more than the fetal adrenal of what was termed CYP11B1 by Incyte Genomics. This is likely to represent almost solely CYP11B1 as the fetal adrenals express very low levels of CYP11B2 (Freije et al. 1997). Transcript levels for CYP21 were similar between fetal and adult adrenals. Interestingly, of the enzymes involved in adrenal steroid hormone production, $3 \beta \mathrm{HSD}$ exhibited the largest difference in expression between the adult and fetal adrenals (24 $\pm 7 \cdot 9$-fold higher in the adult). These data confirm experiments that have demonstrated low levels of $3 \beta \mathrm{HSD}$ observed in HFA using Western and Northern analysis (Doody et al. 1990, Voutilainen et al. 1991). Production of aldosterone and cortisol in the adult adrenal requires the expression of $3 \beta \mathrm{HSD}$ but this enzyme is not necessary for the production of DHEA. Therefore, the lack of $3 \beta \mathrm{HSD}$ expression in the HFA allows substrate to be used for DHEA production.

Of the steroid-metabolizing enzymes, the HFA had higher levels of CYP11A1 (cholesterol side-chain cleav- age) $(2 \cdot 8 \pm 0 \cdot 3$-fold $)$ compared with the adult adrenal. In addition, the steroidogenic acute regulatory protein transcript was elevated by $1.9 \pm 0 \cdot 2$-fold in the HFA. This suggests that the HFA exhibits an increased capacity to produce pregnenolone compared with the adult adrenal. DHEA production also relies on CYP17, cytochrome b5 and $\mathrm{P} 450$-oxidoreductase, which work together to regulate $17 \alpha$ hydroxylase/17,20 lyase activities. Interestingly, there were no differences in the expression of transcripts for CYP17 (17á hydroxylase) or cytochrome b5 and only a modest increase in P450-oxidoreductase $(1.56 \pm 0.13)$ in the HFA vs adult adrenal.

As seen in Table 1, expression of several transcripts for enzymes involved in de novo cholesterol biosynthesis were increased in the HFA. We have previously reported that the HFA has the greatest ability to produce cholesterol of all fetal tissues (Carr \& Simpson 1982). In addition, there was an elevation in the expression of low density lipoprotein (LDL) receptor $(2 \cdot 2 \pm 0 \cdot 2$-fold). The elevated expression of both the enzymes needed for sterol biosynthesis and the LDL receptor suggests that the supply of circulating cholesterol is limiting for the huge amount of steroid being produced by the adrenals during fetal life. A 
Table 2 Transcripts expressed at $\geq 2 \cdot 5$-fold higher levels in adult vs fetal adrenals

Genes

Function

Signal

transduction

Cellular

immunity

Sterol, steroid

metabolism

Growth and

development

Localized and

structural

protein

Transcription

factors

Other
Regulator of G-protein signaling 1

Regulator of G-protein signaling 2, $24 \mathrm{kDa}$

GTP-binding protein overexpressed in skeletal muscle

Complement component 3

CD74 antigen (class II antigen-associated)

Complement component 1, $r$ subcomponent

SB class II histocompatibility antigen $\alpha$-chain

CD163 antigen

$3 \beta \mathrm{HSD}$

Early growth response 1

Nephroblastoma overexpressed gene

Transducer of ERBB2,1

Matrix Gla protein

Actin-binding LIM protein

Transgelin

Lumican

Homo sapiens homeobox protein (HOX-1·3 gene)

Nuclear receptor NGFIB designated NR4A1

EST (uncharacterized transcript)

$v$-fos murine osteosarcoma viral oncogene homolog

v-jun avian sarcoma virus oncogene homolog

ell-related RNA polymerase II, elongation factor

Basic helix-loop-helix domain containing, class B, 2

cAMP responsive element binding protein-like 2

CCAAT-binding transcriptional factor

Aldo-keto reductase family 1 , member B1

KIAA0751 (uncharacterized transcript)

ATP-binding cassette, sub-family B (MDR/TAP), member 1

Putative Ac-like transposon

\section{Fold difference}

Accession no.

\begin{tabular}{rrr}
\hline Mean & & S.E. \\
& & \\
$6 \cdot 6$ & & $1 \cdot 4$ \\
$5 \cdot 4$ & & $1 \cdot 0$ \\
$4 \cdot 2$ & & $1 \cdot 0$ \\
$14 \cdot 0$ & & $5 \cdot 0$ \\
$5 \cdot 7$ & & $1 \cdot 4$ \\
$4 \cdot 1$ & & $1 \cdot 0$ \\
$3 \cdot 4$ & & $0 \cdot 5$ \\
$4 \cdot 1$ & & $1 \cdot 0$ \\
$24 \cdot 0$ & & $7 \cdot 9$
\end{tabular}

M38180

Al083849

X78351

D38305

AW631281

NM_006720

AF013711

AL036211

M26679

NM_002135

Al913832

AW073373

A1078377

U88629

NM_003670

AF039081

X12492

J05474

AA476826

M29447

Al031658
$1 \cdot 4$

$1 \cdot 0$

$\cdot 0$

$5 \cdot 0$

$\cdot 4$

$1 \cdot 0$

$7 \cdot 9$

$3 \cdot 3$ *

$0 \cdot 2$

$0 \cdot 3$

$1 \cdot 1$

$1 \cdot 9$ *

$1 \cdot 4$

$0 \cdot 1$

$1 \cdot 1$

$2 \cdot 8^{*}$

1.9

$1 \cdot 1$

$1 \cdot 8^{*}$

$1 \cdot 4^{*}$

0.5

$0 \cdot 1$

$0 \cdot 1$

0.5

0.5

$0 \cdot 2$

$0 \cdot 3$

*Indicates circumstances where the mean activity of the gene in the fetal adrenal fell between the 95th and the 99th percentile confidence interval of that in the adult adrenal. All others fell outside the 99th percentile confidence interval for the adult adrenal.

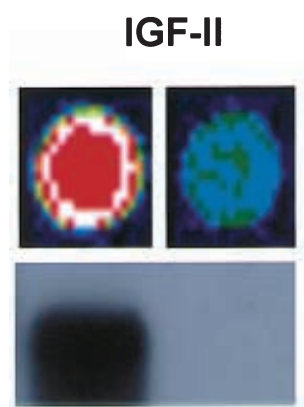

Fetal Adult
KIAA0018
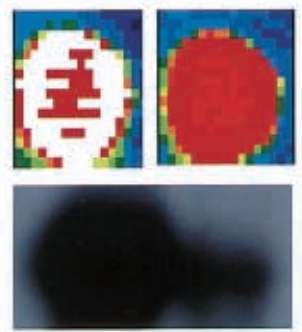

Fetal
Adult

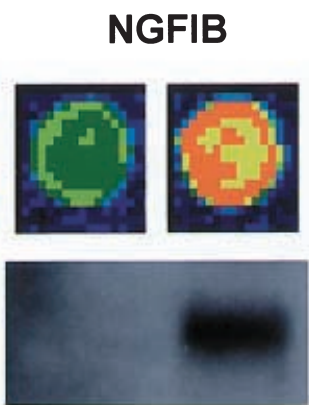

Fetal

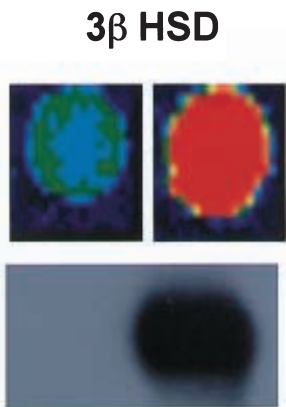

Fetal Adult
- Signal +

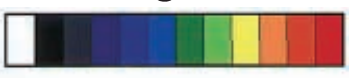

Microarray

Northern

Figure 2 Microarray versus Northern analysis for IGF-II and 3ßHSD. The microarray false color images for IGF-II, KIAA0018, NGFIB and $3 \beta \mathrm{HSD}$ transcript levels for HFA and adult adrenals are shown in the upper panel. The color of the false color image represents gene expression in the sample, i.e. red-orange is more intense while blue-green is less intense. Northern analysis using total RNA from three pooled HFA and three pooled adult adrenals is shown in the lower panel. 
number of studies support the idea that the HFA rapidly metabolizes any available circulating LDL to provide cholesterol for steroidogenesis. These include the observations that fetal circulating LDL is elevated when dexamethasone is given to mothers (Parker et al. 1987) and in anencephalic fetuses that have impaired adrenal function (Parker et al. 1983). In both cases, the level of circulating cholesterol and LDL is significantly higher compared with that seen in normal fetuses. The adult adrenal would appear to have adequate cholesterol supply from circulating LDL. Only under pathologic conditions, as is seen in certain adrenal tumors, does the post-natal adrenal require such a large amount of circulating cholesterol for steroid biosynthesis (Leichter \& Daughaday 1974, Nakagawa et al. 1995). Under these conditions, adrenal tumor production of steroid hormone can be greatly elevated causing hypocholesterolemia, as is seen in the fetus. Thus, in the HFA, the elevated expression of enzymes needed for cholesterol biosynthesis is likely to result from the unique requirement of large amounts of substrate for steroid production.

The size of the HFA (similar to the kidney at 18 weeks) has caused investigators to search for altered growth factor expression that might regulate its hyperplasia. In addition, growth factors have been studied as potential paracrine regulators of steroidogenic capacity of the HFA. The majority of genes categorized as related to growth and development were expressed to a greater degree in the fetal adrenal, with the IGF-II gene being expressed at 25-fold greater levels in the HFA than in the adult adrenal (Table 1). The enhanced HFA expression of IGF-II transcripts (second only to the liver) is well documented (Han et al. 1988). The fact that adrenal IGF-II expression drops dramatically after birth led to the hypothesis that IGF-II could play an important role in the unique functions and/or hyperplasia of the fetal adrenal gland (Mesiano \& Jaffe 1997). In vitro studies have shown that IGF-II, acting through the IGF-I receptor, increases expression of the enzymes in steroid hormone biosynthesis (Lallemand et al. 1996, Mesiano et al. 1997). An important advantage for microarray analysis is the ability to examine the relative expression of several members of the IGF/ insulin system simultaneously. No difference was observed for the expression of insulin receptor or IGF-binding proteins $-1,-3,-4$ or -7 (data not shown). However, the fetal adrenal did exhibit increased expression of IGF-I receptor $(2 \cdot 1 \pm 0 \cdot 04$-fold greater than the adult adrenal). Thus, the fetal adrenal has evolved to produce IGF-II and have increased IGF sensitivity by increasing IGF-I receptors.

In summary, we have used cDNA microarray technology to analyze global differences in gene expression between the human fetal and adult adrenal glands. We have identified 69 transcripts that are differentially expressed by at least $2 \cdot 5$-fold. Importantly, the vast majority of these genes have not been studied with regard to fetal or adult adrenal function.

\section{Acknowledgements}

The authors wish to thank Mary Kaye McDonald and Louella Hupp for their excellent editorial assistance and Bobbie Mayhew, Su Yin and Ming Jian for their expert technical support. This work was supported by awards from the National Institute of Health (DK43140 and HD11149 to W E R) and the Office of Naval Research (to C R P).

\section{References}

Carr BR \& Simpson ER 1982 Cholesterol synthesis in human fetal tissues. Journal of Clinical Endocrinology and Metabolism 55 447-452.

Davis IJ \& Lau LF 1994 Endocrine and neurogenic regulation of the orphan nuclear receptors Nur77 and Nurr-1 in the adrenal glands. Molecular Cellular Biology 14 3469-3483.

Doody KM, Carr BR, Rainey WE, Byrd W, Murry BA, Strickler RC, Thomas JL \& Mason JI 19903 Beta-hydroxysteroid dehydrogenase/isomerase in the fetal zone and neocortex of the human fetal adrenal gland. Endocrinology 126 2487-2492.

Freije WA, Pezzi V, Arici A, Carr BR \& Rainey WE 1997 Expression of 11 -hydroxylase (CYP11B1) and aldosterone synthase (CYP11B2) in the human fetal adrenal. Journal of the Society of Gynecologic Investigation 4 305-309.

Greeve I, Hermans-Borgmeyer I, Brellinger C, Kasper D, Gomez-Isla T, Behl C, Levkau B \& Nitsch RM 2000 The human DIMINUTO/DWARF1 homolog seladin-1 confers resistance to Alzheimer's disease-associated neurodegeneration and oxidative stress. Journal of Neuroscience 20 7345-7352.

Han VK, Lund PK, Lee DC \& D'Ercole AJ 1988 Expression of somatomedin/insulin-like growth factor messenger ribonucleic acids in the human fetus: identification, characterization, and tissue distribution. Journal of Clinical Endocrinology and Metabolism 66 422-429.

Klahre U, Noguchi T, Fujioka S, Takatsuto S, Okota T, Nomura T, Yoshida S \& Chua N 1998 The arabidopsis DIMINUTO/ DWARF1 gene encodes a protein involved in steroid synthesis. Plant Cell 10 1677-1690.

Lallemand D, Penhoat A, Lebrethon MC, Ardevol R, Baehr V, Oelkers W \& Saez JM 1996 Insulin-like growth factors enhance steroidogenic enzyme and corticotropin receptor mRNA levels and corticotropin steroidogenic responsiveness in cultured human adrenocortical cells. Journal of Clinical Endocrinology and Metabolism 81 3892-3897.

Leichter SB \& Daughaday WH 1974 Massive steroid excretion and hypocholesterolemia with an adrenal adenoma. Report of a case. Annals of Internal Medicine 81 638-640.

Mesiano S \& Jaffe RB 1997 Developmental and functional biology of the primate fetal adrenal cortex. Endocrine Reviews 18 378-403.

Mesiano S, Mellon SH \& Jaffe RB 1993 Mitogenic action, regulation, and localization of insulin-like growth factors in the human fetal adrenal gland. Journal of Clinical Endocrinology and Metabolism 76 968-976.

Mesiano S, Katz SL, Lee JY \& Jaffe RB 1997 Insulin-like growth factors augment steroid production and expression of steroidogenic enzymes in human fetal adrenal cortical cells: implications for adrenal androgen regulation. Journal of Clinical Endocrinology and Metabolism 82 1390-1396.

Nakagawa T, Ueyama Y, Nozaki S, Yamashita S, Menju M, Funahashi T, Kameda-Takemura K, Kubo M, Tokunaga K, Tanaka T et al. 1995 Marked hypocholesterolemia in a case with adrenal adenoma-enhanced catabolism of low density lipoprotein (LDL) via the LDL receptors of tumor cells. Journal of Clinical Endocrinology and Metabolism 80 92-96. 
Parker CR Jr, Carr BR, Winkel CA, Casey ML, Simpson ER \& MacDonald PC 1983 Hypercholesterolemia due to elevated low density lipoprotein-cholesterol in newborns with anencephaly and adrenal atrophy. Journal of Clinical Endocrinology and Metabolism $\mathbf{5 7}$ 37-43.

Parker CR Jr, MacDonald PC, Carr BR \& Morrison JC 1987 The effects of dexamethasone and anencephaly on newborn serum levels of apolipoprotein A-1. Journal of Clinincal Endocrinology and Metabolism 65 1098-1101.

Voutilainen R, Ilvesmaki V \& Miettinen PJ 1991 Low expression of $3 \beta$-hydroxy-5-ene steroid dehydrogenase gene in human fetal adrenals in vivo: adrenocorticotropin and protein kinase C-dependent regulation in adrenocortical cultures. Journal of Clinical Endocrinology and Metabolism 72 761-767.

Wilson TE, Mouw AR, Weaver CA, Milbrandt J \& Parker KL 1993 The orphan nuclear receptor NGFI-B regulates expression of the gene encoding steroid 21-hydroxylase. Molecular and Cellular Biology $13861-868$.

Received 25 May 2001

Accepted 11 July 2001 